\title{
FEATURES OF CHANGES OF STRUCTURE AND ADHESIVE PROPERTIES FOR ACRYLIC ADHESIVES UNDER THE INFLUENCE OF THE FILLER
}

\author{
Alla Garbuz \\ Department of structural and theoretical mechanics \\ O. M. Beketov Kharkiv National University of Municipal Economy \\ 12 Revolution str, Kharkov, Ukraine, 61000 \\ alla-garbuz@mail.ru \\ Pavel Bilym \\ Department of Labour Protection \\ O. M. Beketov Kharkiv National University of Municipal Economy \\ 12 Revolution str, Kharkov, Ukraine, 61000 \\ Pashha@mail.ru \\ Denis Zubenko \\ Department of electric transport \\ O. M. Beketov Kharkiv National University of Municipal Economy \\ 12 Revolution str, Kharkov, Ukraine, 61000 \\ Denis04@ukr.net
}

\begin{abstract}
The research results of the effect of concentration of injected mineral filler on change in adhesion strength of acrylic adhesive composition to the metal substrate are given in the article. The adhesive composition was prepared based on pre-compounded reaction system: the stabilized monomer - methyl methacrylate and polymer modifier - sterol copolymer with methyl methacrylate with followed filling and polymerization in the presence of a redox system.

It is shown that the mobilization to resource preservation of the adhesive contact at the allowable amount of the filler and the thickness of the adhesive layer is due to the overall contribution of the dispersed phase in the change of the structure and macrophysical properties. Experimental data confirm the obtained dependencies: the adhesive strength of the adhesive from the contact area to steel wire; density, glass transition temperature and the calculated value of the Simcha-Boyer constant of block samples from the amount of the filler; character of reducing dynamic shear modulus of the filled composition during heating.

It is suggested that the decrease in density in a volume of polymer phase of filled system provides a slight decrease in adhesion strength of acrylic adhesive to the substrate.

Keywords: acrylic adhesive, filler, adhesive strength, macrophysical properties, free volume of the polymer phase.

DOI: $10.21303 / 2461-4262.2016 .00075$

\section{Introduction}

Polymer adhesives based on reactive acrylic compounds are proven in the modern industrial practice of building production [1]. However, to make the necessary workability when connecting (bonding) of dissimilar materials and the implementation of acceptable performance characteristics of the adhesive joint of construction structural elements it have to deal with the additional injection of mineral fillers in the polymer matrix [2].

It is known that surfaces of inorganic fillers have a high adsorption activity. Molecules and atoms that adsorbed on the surface create new surface states [3] and the energy spectrum of the surface largely determines the nature of physical and chemical interaction of the polymer - filler. Structure and properties of the boundary layer depends largely on the nature and surface topography of the substrate and can vary from the structure and properties of the polymer in the bulk adhesive film. Therefore, the adhesive contact strength of the disperse-filled polymer with the substrate varies in a complicated manner and is generally dependent on the nature, size of the filler particles and its content in the polymer phase [4].

Due to the nature to the structuring of filled acrylic systems in this work tasked to study the action of mineral filler and its amount for the implementation of the adhesion strength of the metal substrate.
\end{abstract}

(C) Alla Garbuz, Pavel Bilym, Denis Zubenko 
The study of the polymerization of styrene and methyl methacrylate, copolymerization of styrene with divinylbenzene in the presence of mineral fillers with a different composition shows the effect of the latter on the polymer structurization [5]. The development of these studies, which are critical for the choice of formulation for adhesive filled systems, is established adhesive and elastic properties, volumetric thermal expansion coefficient, glass transition temperature and density of the methyl methacrylate-based polymer adhesive, polymerized in the presence of mineral filler.

\section{Materials and methods}

The object of this article was prepolymer based on stabilized methyl methacrylate and polymer modifier - styrene copolymer with methyl methacrylate, taken at a joint compounding in the weight ratio of 100:20 [6]. The fine grinding quartz send $(\mathrm{d}=30-50 \mathrm{~m})$ was selected as the filler, without special pre-cleaning, the amount of which was varied from 10 to $50 \mathrm{wt}$. $\%$ on $100 \mathrm{wt}$. \% of prepolymer. Initiation for the final structuring of filled and unfilled adhesive was carried out by redox system: benzoyl peroxide $-\mathrm{N}, \mathrm{N}$ 'dimethyl aniline.

Test samples were produced by casting in open molds and cups (for adhesion test) and hardened (polymerized) at room temperature for 24 hours.

Dynamic mechanical testing of the experimental block samples performed in forced resonant oscillating of cantilever fixed sample at frequencies of 20-200 Hz with simultaneous recording of linear extension of the sample at a heating rate in the heat chamber of 2 degree/min. The adhesion strength of the polymer adhesive - steel wire was determined on the single-cavity adhesion tester under normal conditions [7]. Density of the block samples was determined by weighing in distilled water [8]. Density of the polymer adhesive (resin) in the filled material was calculated from the additivity rule [9].

\section{Discussion of the results}

As can be seen from the data presented in Fig. 1, decrease of adhesion strength with increasing contact area (depending on the cup height) is common phenomenon: it is observed for the compounds prepared at various concentrations of the filler and in the absence thereof. There are three possible explanations of this dependence [7]:

- effect of the known statistic scale factor, i.e. increasing the probability of occurrence of a dangerous defect with increasing sample sizes;

- non-uniform distribution of shear (tangential) stresses occurring at the interface between steel wire - adhesive upon application of an external load;

- residual "internal" stress occurring at the interface with the formation of compounds.

Meanwhile, it should be noted the two observed in Fig. 1 features:

- injection of the filler leads to a sharp decrease in adhesion strength;

- curves circuit has a characteristic "fan-shaped" view - with a small contact area curves diverge, and at large - agree. The exceptions are the curves 5 and 6 (Fig. 1), which correspond to the higher content of the filler and do not fit into the general ensemble of presented dependence.

The latter fact suggests that the pulverized quartz sand in the acrylic system is not a "pure" extender, i. e. its effect is not limited to ballast in a polymer matrix with an acceptable amount.

Based on the data presented in Fig. 2, density of the acrylic adhesive, polymerized in the presence of increasing amounts of filler falls. Moreover, the decrease in density is more rapidly with an initial amount of the filler.

Simcha-Boyer coefficient (K1) was calculated on the basis of experimental data obtained by linear dilatometry (registration of linear extension for block sample) using the formula:

$$
\mathrm{K}_{1}=\mathrm{T}_{\mathrm{GL}}\left(\beta_{\mathrm{R}}-\beta_{\mathrm{GL}}\right),
$$

where $\beta_{\mathrm{R}}$ and $\beta_{\mathrm{GL}}$ - volumetric thermal expansion coefficients of the adhesive in the rubbery and glassy state, respectively, $\mathrm{T}_{\mathrm{GL}}$ - structural glass transition temperature.

It should be noted that this coefficient characterizes a free volume fraction in the glass transition temperature. According to the literature data [10] for many polymers regardless of their chemical composition the coefficient $\mathrm{K} 1$ is constant and equal to 0.113 . However, as can be seen from the data 
presented in Fig. 2 (curve 2), the value of coefficient K1, and hence the free volume fraction at glass transition temperature of the resin increases with increasing degree of filling.

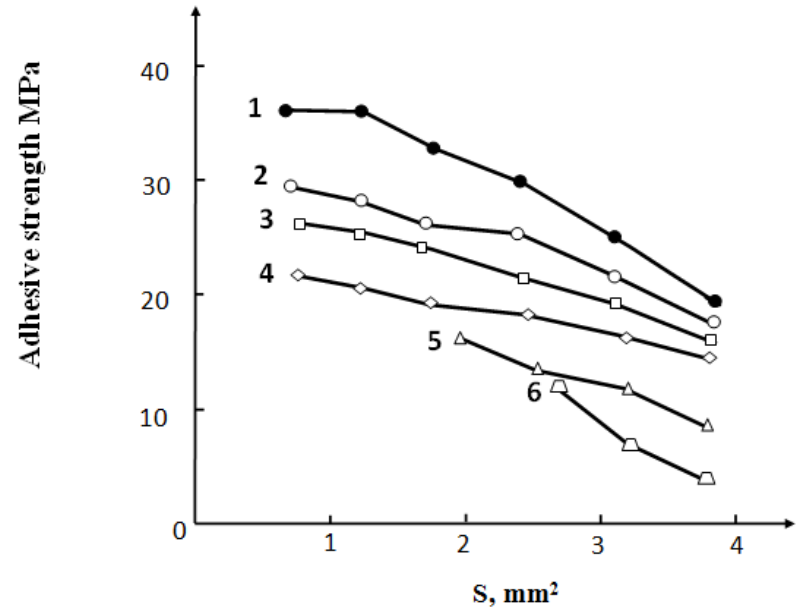

Fig. 1. Dependence of the adhesive strength on the contact area of the system a steel wire with a diameter of $200 \mathrm{~mm}$ - polymer adhesive: acrylic unfilled part - 1; the filler content in the polyacrylate $10,20,30,40,50 \mathrm{wt}$. $\%$ - curves $2,3,4,5$ and 6 , respectively

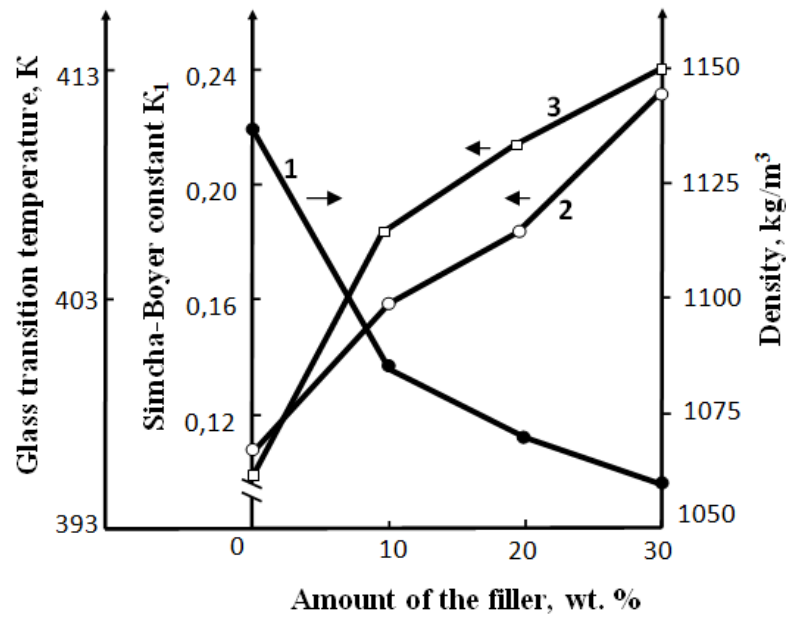

Fig. 2. Dependence of the density at $25^{\circ} \mathrm{C}$ of Simcha-Boyer constant and glass transition temperature on the filler concentration: density of acrylic resin in composition with quartz - 1 ; Simcha-Boyer constant for acrylic resin in composition with quartz - 2 and the temperature of the mechanical glass transition of acrylic composition with quartz

Such diverse effect of the filler on acrylate polymerization process - increasing the glass transition temperature and at the same time decrease of the density and increase of the free volume in the "hardened" resin with increase the proportion of the filler in the material - largely explains the results of determination of the elastic modulus of filled systems at different temperatures (Fig. 3).

Thus, the elastic modulus (dynamic shear modulus) of the filled systems decreases with increasing temperature is much more intense than unfilled polymer resin and the more than the higher the degree of filling. Hence, quartz sand is an intense adsorbent of polymer molecules, in particular due to the orientation of the ester groups of the acrylic polymer to the filler surface [4]. Therefore hardening resin molecules in the adsorbed layer promotes more increase of elastic modulus of the system and glass transition temperature, than the more polymer macromolecules involved in the boundary layer to the filler, that is, with increasing filler concentration. 


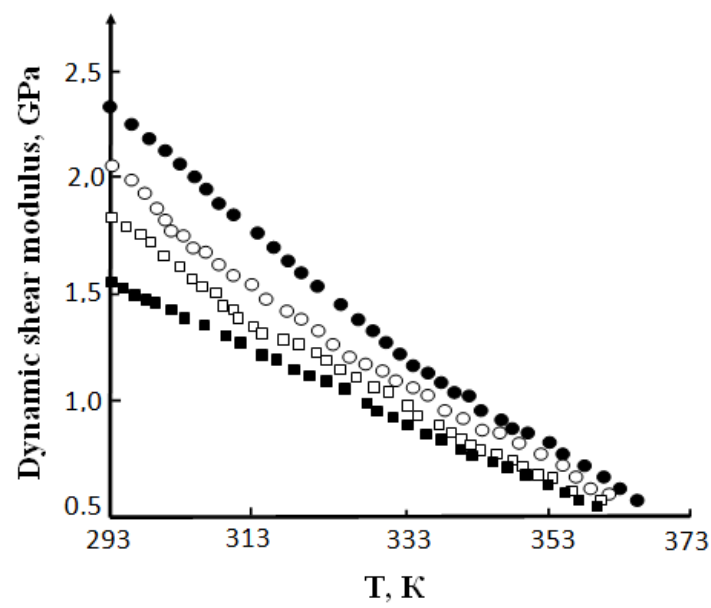

Fig. 3. Dependence of the dynamic shear modulus of the acrylic composition with quartz on temperature: the filler content - 10 wt. $\%$ - ; 20 wt. $\%$ - ०; 30 wt. $\%$ - •; unfilled -

At the same time, no matter how tightly packed adsorption layer, its density should be lower than in the matrix (resin) phase where the polymerization process was the same intensity as acrylic system samples containing no filler. Density and deformability of the adsorption layer should decrease with increasing temperature (Fig. 3), causing a greater decrease in the modulus of elasticity that characterizes the total number of physical nodes (weaves) of polymer network.

\section{Conclusions}

1. At injection of fine grinding quartz send into an acrylic system supramolecular structures are formed with a characteristic surface layer, which makes a significant contribution to changing macrophysical characteristics of the material. Therefore, mobilization to resource preservation of adhesive contact with acceptable amounts of the filler (30 wt. \% in the polyacrylate) and the thickness of the adhesive layer (Fig. 1) is due to the overall contribution of the dispersed phase in the hardening polymer.

2. Overlay of the various factors, primarily, density decrease of polymer phase in a filled system should ensure minimal shrinkage during polymerization, which promotes a slight decrease of adhesive strength of the acrylic adhesive to the metal substrate.

\section{References}

[1] Zolotov, M. S., Shutenko, L. N., Psurtseva, N. A., Dushyn, V. V. (1985). Experience of adhesive joints in construction. Kharkiv: KhIIKS, 19-98.

[2] Garbuz, S. A. (1998). Anchor connection to acrylic adhesives with high adhesive strength. The use of plastics in construction and municipal services: Materials IV Ukr. Scientific and Engineering. Conf. Kharkiv: KhNAME, 12-14.

[3] Kiselev, V. F., Krylov, O. V. (1980). Electronic phenomena in adsorption and catalysis on semiconductors and dielectrics. Moscow: Science, 234.

[4] Kerber, M. L., Vinogradov, V. M., Golovkin, G. S. et al.; In: Berlin, A. A. (2008). Polymeric composites: structure, properties, technology. St. Petersburg: Profession, 560.

[5] Lipatov, Y. S. (1977). Physical chemistry of filled polymers. Moscow: Chemistry, 304.

[6] Rivat-Lahousse, A. (1956). Les colles industrielles. Paris: Edite par Dunod, 432.

[7] Gorbatkina, Y. A. (1987). The adhesion to the polymer fiber. Moscow: Chemistry, 192.

[8] Koenig, G. (Ed.) (1982). The latest instrumental methods of studying the structure of polymers. Moscow: Mir, 264.

[9] Katz, H. S., Milewski, J. V. (1981). Handbook of fillers and reinforcements for plastics. Translation from English. Moscow: Chemistry, 736.

[10] Simha, R., Boyer, R. F. (1962). On a General Relation Involving the Glass Temperature and Coefficients of Expansion of Polymers. The Journal of Chemical Physics, 37 (5), 1003. doi: 10.1063/1.1733201 